\title{
An Optimal Topology-Transparent Scheduling Method in Multihop Packet Radio Networks
}

\author{
Ji-Her Ju, Member, IEEE, and Victor O. K. Li, Fellow, IEEE
}

\begin{abstract}
Many transmission scheduling algorithms have been proposed to maximize the spatial reuse and minimize the timedivision multiple-access (TDMA) frame length in multihop packet radio networks. Almost all existing algorithms assume exact network topology information and do not adapt to different traffic requirements. Chlamtac and Farago proposed a topologytransparent algorithm. Following their approach, but with a different design strategy, we propose another algorithm which is optimal in that it maximizes the minimum throughput. We compare our algorithm with that of Chlamtac and Farago's and with the TDMA algorithm, and find that it gives better performance in terms of minimum throughput and minimum and maximum delay times. Our algorithm requires estimated values of the number of nodes and the maximum nodal degree in the network. However, we show that the performance of our algorithm is insensitive to these design parameters.
\end{abstract}

Index Terms - Packet radio network, time-division multiple access, topology-transparent scheduling.

\section{INTRODUCTION}

A PACKET radio network consists of a number of geographically dispersed radio units which communicate with each other. Due to limited transmission power, it may be necessary to relay a packet over multiple nodes before the destination is reached, resulting in a multihop packet radio network. A packet radio network using a time-division multiple-access (TDMA) transmission schedule is called a TDMA network. In a conventional TDMA network every node is assigned a unique time slot in each frame to transmit. This method works well when the network is fully connected, but in a multihop packet radio network the number of nodes in the network is much greater than the maximum number of neighbors of a node, and spatial reuse of time slots can greatly improve the system performance. Hence, the design of transmission schedules in multihop mobile radio networks to ensure good system performance is an attractive research topic [1], [2], [4], [5], [7], [15], [16]. A proper design not only guarantees successful information exchanges among nodes in the presence of conflicts but also maximizes the system throughput. In this context we will focus on the scheduling problem in a TDMA network, although our algorithm can also be applied to a code-division multiple-

Manuscript received February 10, 1995; revised November 14, 1995 and March 1, 1997; approved by IEEE/ACM TRANSACTIONS ON NETWORKING Editor I. Cidon.

J.-H. Ju is with the Chung Shan Institute of Science and Technology, Taiwan, R.O.C.

V. O. K. Li is with the Department of Electrical and Electronic Engineering, The University of Hong Kong, Hong Kong (e-mail: vli@eee.hku.hk).

Publisher Item Identifier S 1063-6692(98)03565-1. access (CDMA) network using a procedure similar to that described in [3]. In a TDMA system, time is divided into transmission slots, grouped into frames. Each slot is designed to accommodate the transmission of one fixed-size packet and a guard time, corresponding to the maximum differential propagation delay between pairs of nodes in the network. When nodes communicate, they may suffer two types of conflicts [7], [12]. The first one, called primary conflict, occurs if two or more nodes transmit simultaneously to the same destination node. The second one, called secondary conflict, occurs when a node receiving a transmission is also within the transmission range of other transmissions not intended for it.

Previous studies on transmission scheduling can be classified into two categories: link activation [1], [9] and node activation [7], [15]. Most such studies concentrated on finding fair conflict-free algorithms which maximize the system throughput by using graph theory [9], [16]. In [7] it is shown that the problem of determining transmission schedules with optimal throughput is NP-complete. The problem of constructing a minimum frame length schedule is also shown to be NP-complete [15]. Most algorithms are centralized, i.e., they need global network connectivity information to achieve their goals. Ephremides and Truong [7] proposed a distributed algorithm which requires using up to two-hop connectivity information. The distributed algorithm of Ramaswami and Parhi [15] only needs one-hop connectivity information. As can be expected, however, an optimal design for an initial network may be far from optimal after changes in the network topological structure or the traffic load of the nodes. In particular, in a highly mobile network, accurate network connectivity information may be very difficult to obtain. Furthermore, to maintain accurate connectivity information will require a great number of information exchanges among the nodes. This intensive communication requirement may consume more bandwidth than the savings obtainable by an algorithm which uses this more accurate topology information. Therefore, the efficiency and robustness of the above algorithms are questionable in mobile networks. By allowing contentions, Chlamtac and Farago [2] developed an algorithm which is topology-transparent and guarantees that each node has at least one successful transmission in each frame. However, no attempt has been made to optimize the performance of this algorithm. In fact, as will be shown in Section V, this algorithm sometimes performs worse than a conventional TDMA algorithm. Following their approach, but with a different design strategy, we propose another algorithm which is optimal in that it maximizes the minimum throughput. 
We compare our algorithm with that of Chlamtac and Farago's and with the TDMA algorithm, and find that it gives better performance in terms of minimum throughput and minimum and maximum delay times. Our algorithm requires estimated values of the number of nodes and the maximum nodal degree in the network. However, we show that the performance of our algorithm is insensitive to these design parameters.

In this paper we develop a new link activation scheduling algorithm with a minimum guaranteed throughput under any traffic conditions and analyze its performance for pointto-point packet transmissions. The rest of this paper is organized as follow. In Section II we present our network model and some definitions and theorems which will be used in the following sections. Section III presents the steps of our proposed algorithm. Some theorems related to the choice of the optimal frame structure and the system parameters are also given. In Section IV the average maximum and minimum delay times are derived and an analytical comparison between our algorithm and the conventional TDMA algorithm is provided. Numerical results and discussions comparing our optimal algorithm, Chlamtac and Farago's algorithm, and the conventional TDMA algorithm are given in Section V. We conclude in Section VI.

\section{Model AND Definitions}

A multihop packet radio network with $N$ mobile nodes can be represented by a directed graph $G(V, E) . V$ is the set of all network nodes and $|V|=N . E$ is the set of all edges. If node $v_{j}$ is within the transmission range of $v_{i}$, then an edge denoted $\left(v_{i}, v_{j}\right)$ is in $E$. We assume that if $\left(v_{i}, v_{j}\right) \in E$, then $\left(v_{j}, v_{i}\right) \in E . v_{i}, v_{j}$ are said to be neighbors. The degree of a node $v$, i.e., $D(v)=|\{(u, v) \mid(u, v) \in E ; u \in V\}|$, is defined as the number of its neighbors. The maximum degree $D_{\max }$, i.e., $\max _{v \in V} D(v)$ in the network is much less than the number of nodes in the network. We assume that $D_{\max }$ will remain constant while the network operates. The maintenance and benefits of this kind of degree-bounded topology have been discussed in [10], [11], and [14].

In this paper we assume that the transmission channel is error-free and a reception failure is due only to packet collisions. The packet transmitted from a neighbor of a node, say node $s$, is successfully received by node $s$ if no other neighboring nodes transmit in the same slot. All of the nodes in the network are homogeneous, i.e., they have the same equipment, traffic characteristics, and traffic generation rate. Although nodes with multiple reception capability have been considered in [4] and [8], we assume that each node only has a single narrow-band transceiver to communicate with other nodes. A node cannot transmit and receive simultaneously. As in [2], we will use the total number of nodes $(N)$ and the maximum degree $\left(D_{\max }\right)$ as design parameters. Based on these two system parameters, we will design a scheduling algorithm such that each node in the network gets a predetermined minimum throughput no matter how or how often the topology changes.

Our approach is to use coding theory to design a topologytransparent scheduling algorithm, and to maximize the guar-
TSAF of Node A: $f_{A}(x)=a_{k} x^{k}+a_{k-1} x^{k-1}+\ldots+a_{0} \quad(\bmod p)$ $\begin{array}{llllll}\text { subframe } & 0 & \text { subframe } & 1 & \ldots & \text { subframe }(p-1)\end{array}$

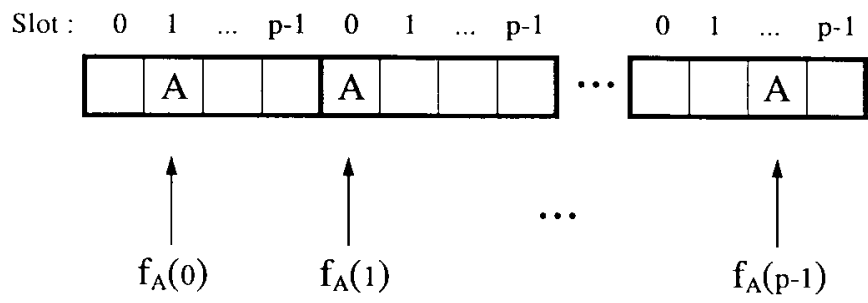

TSLV of Node $A: \quad\left(f_{A}(0), f_{A}(1), \ldots, f_{A}(p-1)\right)$

Fig. 1. The relationships of TSAF, TSLV, and the frame structure.

anteed throughput of our algorithm. The following definitions and theorems help explain our algorithm.

Definition 1: A polynomial $f(x)$ of degree $k \bmod p$ can be expressed as $f(x)=\sum_{i=0}^{k} a_{i} x^{i}(\bmod p)$, where $a_{i} \in$ $\{0,1,2, \cdots, p-1\}$.

Theorem 1: Let $f(x)$ be a polynomial of degree $k \bmod$ $p$, where $p$ is a prime number. Then the equation $f(x)=0$ $(\bmod p)$ will have at most $k$ distinct roots which are integers between 0 and $p-1$.

The proof of the above theorem can be found in [6].

Definition 2: The Hamming weight $H W(X)$ of a $p$-tuple row vector $X$ is the number of nonzero symbols in the vector $X$.

Definition 3: The Hamming distance $H D(X, Y)$ between two $p$-tuple row vectors $X, Y$ is the number of symbols in which the two $p$-tuple row vectors differ.

The Hamming weight and Hamming distance will be used to describe the relationship between the transmission slot assignments of two nodes.

Definition 4: For a given network $G(V, E)$ each node is associated with a unique time slot assignment function (TSAF) $f_{v}(x)=\sum_{i=0}^{k} a_{i} x^{i}(\bmod p), v \in V \cdot f_{v}(x)$ is a polynomial with degree $k \bmod p$ and it maps from $Z_{p}$ to $Z_{p}$, where $Z_{p}=\{0,1,2, \cdots, p-1\}$ and $p$ is a prime.

This TSAF is used to calculate the positions of assigned transmission slots in a frame for each node.

Definition 5: We define a standard row vector $S=$ $(0,1,2, \cdots, p-1)$. Then $f_{v}(\boldsymbol{S}) \equiv\left(f_{v}(0), f_{v}(1), \cdots, f_{v}(p-1)\right)$ is also a row vector called the time slot location vector (TSLV) of node $v$.

This TSLV indicates the exact positions of assigned transmission slots in each frame for each node. In Fig. 1 we show the relationships of TSAF, TSLV, and the frame structure. Each frame is divided into $p$ subframes of $p$ slots each, and the time slot assigned to node $A$ in subframe $i, i=0,1, \cdots, p-1$ is given by $f_{A}(i) \bmod p$. In Fig. $1 f_{A}(0)=1, f_{A}(1)=0$, etc.

The following is used to define the relationship between any two TSAF's.

Definition 6: For a set of TSAF's $f_{v}(x)$ with degree $k$, we say that two TSAF's are in the $m$ th-order TSAF subgroup if the difference of these two TSAF's is a polynomial with degree $m$. 
Theorem 2: Consider a set of TSAF's $f_{v}(x)$ with maximum degree $k$. Let $X=f_{i}(S)$ and $Y=f_{j}(S)$ be two TSAF's, where $S$ is the standard row vector. Then the $H D(X, Y)$ of any $X$ and $Y$ is greater than or equal to $p-m$ if $f_{i}(x)$ and $f_{j}(x)$ are in the $m$ th-order subgroup of the subset of TSAF's $f_{v}(x) . p$ is the dimension of the TSLV and $0 \leq m \leq k$.

Proof: If $f_{i}(x)$ and $f_{j}(x)$ are in the $m$ th-order TSAF subgroup, then $f_{i}(x)-f_{j}(x)$ can be expressed as $f_{i j}(x)=$ $\sum_{l=0}^{m} \quad a_{l} x^{l}(\bmod p)$, where $a_{l}$ are elements in $Z_{p}=$ $\{0,1,2, \cdots, p-1\}$ and $a_{m} \neq 0$. The equation

$$
a_{m} x^{m}+\cdots+a_{0} \equiv 0 \quad(\bmod p), \quad \text { where } a_{m} \in Z_{p}
$$

will have at most $m$ roots. That means $X$ and $Y$ have at most $m$ common values among the $p$ elements. So, $H D(X, Y) \geq$ $(p-m)$ for any $X$ and $Y$.

Since the TSLV's $X$ and $Y$ represent the slot positions of assigned transmission slots, each agreement in these two vectors is a possible collision for these two nodes. Theorem 2 says that the number of possible collisions for two nodes is equal to $m$ when their TSAF's are in the $m$ th-order subgroup.

\section{Optimal Scheduling Algorithm}

We now describe our proposed algorithm. Consider a singlechannel TDMA network with $N$ mobile nodes and maximum degree $D_{\max }$, i.e., the number of neighbors of any node in this network is not greater than $D_{\max }$. The TDMA frame consists of $p$ subframes, each consisting of $p$ slots. Each node will be assigned one transmission slot in each subframe according to its unique TSAF $f_{v}(S)$. This idea is similar to that in Chlamtac and Farago [2]. Their algorithm attempts to find the smallest value of $p$ which guarantees that each node has at least one successful transmission in each frame and at the same time minimizes the frame length. In a contention-free scheduling algorithm a frame structure with minimum frame length can indeed maximize the throughput. However, if contentions are allowed, this is no longer true. Hence, we use a different strategy to derive a different scheduling algorithm which can be proved to have certain advantages. In fact, in terms of maximal minimum throughput, our assignment function is optimal.

As we have mentioned, the assigned time slots for each node can be represented by a TSLV. The TSLV is a row vector which can be represented as $\left(s_{1}, s_{2}, \cdots, s_{p}\right)$. If the elements at the same position of two TSLV's have the same value, a collision may occur when the two corresponding nodes transmit because they are assigned the same slot in the same subframe. This implies that the smaller the value of the maximum Hamming weight $\left(H W_{\max }(X)\right)$ of the difference vector between any two TSLV's, the larger the probability that their transmissions will collide. For example, if the dimension of TSLV is $p$, then the maximal value of $H D_{\min }(X, Y)$ between any two TSLV's, say $X$ and $Y$, will be $p$. If $H D_{\min }(X, Y)=$ $p$ for any two TSLV's, the assigned slots for these two nodes are collision-free because there is no agreement in their TSLV's. Hence, we need to find a set of appropriate TSAF's which can generate TSLV's with maximum Hamming distance subject to the maximal minimum throughput requirement.
Since all TSLV's must be unique to each node to ensure that every node has at least a minimum throughput, the total number of TSLV's $N_{T S L V}$ should be at least the total number of nodes in the network, i.e., $N_{\mathrm{TSLV}} \geq N$. Each TSLV is generated from its unique TSAF $f_{v}(x)=\sum_{i=0}^{k} a_{i} x^{i}(\bmod p)$ where $a_{i} \in Z_{p}$, i.e.,

$$
\begin{aligned}
\mathrm{TSLV}:\left(s_{1}, s_{2}, \cdots, s_{p}\right) & =\left(f_{v}(0), f_{v}(1), \cdots, f_{v}(p-1)\right) \\
& =f_{v}(S)
\end{aligned}
$$

and $S$ is the standard row vector. Hence

$$
N \leq N_{\mathrm{TSLV}}=p^{k+1}
$$

if the maximum degree of the TSAF's is equal to $k$.

If the maximum degree of the TSAF's is equal to $k$, the maximum degree of the difference polynomial of any two TSAF's will be less than or equal to $k$. According to Theorem 1 , there are at most $k$ common roots for these two TSAF's. Each common root corresponds to an agreement in their TSLV's and each agreement represents a possible collision for these two nodes. Hence, the maximum degree of the TSAF's $k$ determines the maximum number of possible collisions that a node can cause its neighbor. Based on Theorem 2 we conclude that the number of possible collisions in each frame for any two nodes is between 0 and $k$, and this number of possible collisions depends on the degree of their difference polynomial. Because each node is assigned $p$ transmission slots in each frame and the maximum number of neighbors of a node is $D_{\max }$, the maximum number of possible collisions of a node is $k D_{\max }$. To ensure that each node has some minimum throughput, the following relation must be satisfied:

$$
\begin{aligned}
& p \geq T=p-(\text { number of collisions in a frame }) \\
& \quad \geq\left(p-k D_{\max }\right)>0 .
\end{aligned}
$$

Here, $T$ is the number of guaranteed successful transmissions in each frame for every node. $T_{\max }$ and $T_{\min }$ are the upper and lower bounds of $T$ which are equal to $p$ and $p-k D_{\max }$, respectively.

To evaluate the performance, we define the minimum throughput $\left(G_{\min }\right)$ as follows.

Definition 7: The minimum throughput $\left(G_{\min }\right)$ is defined as the ratio of the number of guaranteed successful transmissions in each frame to the frame length $L$, i.e.,

$$
G_{\min }=T_{\min } / L
$$

where $L=p^{2}$.

Theorem 3: For a given $k$, the maximal value (i.e., upper bound) of $G_{\min }$ is

$$
\max \left(G_{\min }\right)=\left\{\begin{array}{c}
1 /\left(4 k D_{\max }\right), \\
\quad \text { if } N^{1 /(k+1)} \leq 2 k D_{\max } \\
\left(N^{1 /(k+1)}-k D_{\max }\right) /\left(N^{2 /(k+1)}\right), \\
\quad \text { otherwise. }
\end{array}\right.
$$


Proof: From (5), we know

$$
G_{\min }=T_{\min } / L=\left(p-k D_{\max }\right) / p^{2} .
$$

To find the maximal value of $G_{\min }$ we have to solve the following equation:

$$
\begin{aligned}
\frac{\partial G_{\min }}{\partial p} & =0 \\
2 k D_{\max } p^{-3}-p^{-2} & =0 \\
p^{-3}\left(2 k D_{\max }-p\right) & =0 .
\end{aligned}
$$

Since $p \geq k D_{\max } \geq 0$, we get

$$
p=2 k D_{\max } \text {. }
$$

$G_{\min }$ increases with $p$ when $k D_{\max } \leq p \leq 2 k D_{\max }$ and decreases with $p$ when $p \geq 2 k D_{\max }$. From (3) and (4), we know that $p$ has to satisfy the following equations:

$$
\begin{aligned}
& p \geq N^{1 /(k+1)} \\
& p \geq k D_{\max } .
\end{aligned}
$$

Hence, if $N^{1 /(k+1)} \leq 2 k D_{\max }, G_{\min }$ assumes the maximal value when $p=2 k \bar{D}_{\max }$, and

$\max \left(G_{\min }\right)=\left(2 k D_{\max }-k D_{\max }\right) /\left(2 k D_{\max }\right)^{2}=1 /\left(4 k D_{\max }\right)$.

Otherwise, $G_{\min }$ has the maximal value when $p=N^{1 /(k+1)}$, and

$$
\max \left(G_{\min }\right)=\left(N^{1 /(k+1)}-k D_{\max }\right) /\left(N^{2 /(k+1)}\right) .
$$

To maximize the minimum throughput of the network, we have to choose the best values of $k$ and $p$ by using Theorem 3 . Once we decide the values for $k$ and $p$, we can get $N_{\mathrm{TSLV}}=p^{k+1}$ and $T_{\min }=p-k D_{\max }$. In our analysis we found that the best value of $k$ will be greater than one only when $D_{\max } \leq 0.1464 N^{1 / 2}$. For example, if $N=1024$, then $k$ will be equal to two when $D_{\max } \leq 5$. This means that the maximum number of collisions between any two nodes in a frame will be either zero or one in almost all cases if the network size is not extremely large. This result means that the best set of TSLV's are constructed by the TSLV's with $H D(X, Y) \leq(p-1)$. The actual maximum number of collisions of a node depends on the relationships between its TSAF and its neighbors' TSAF's. Note that $k=0$ corresponds to the conventional TDMA fixed time slot assignment algorithm which assigns each node a unique transmission slot in each frame, and the minimum throughput is equal to $1 / N$.

Thus, we can design an optimal scheduling algorithm as below.

\section{Optimal Algorithm:}

- Use Theorem 3 to select the $k$ and $p$ for the given $N$ and $D_{\max }$ such that $G_{\min }$ is maximized.

- Each node is randomly assigned a unique TSAF (with degree $\leq k$ ).

- Each node calculates its TSLV according to (2).

- Each node transmits its data packets at its assigned slots.
Here, we assign the TSAF to each node randomly. Actually, if we have additional information on the nodes' locations, a more systematic way to assign the TSAF's may improve the overall system performance. For example, we can assign the TSAF's from the zeroth-order TSAF subgroup to those nodes in the dense area of the network, i.e., those nodes with many neighbors. This is because the TSAF's from the zeroth-order TSAF subgroup always have the largest $H D_{\min }$.

\section{PeRformance AnAlysis}

In the following analysis the average transmission delay is defined as the average waiting time between two successive successful transmissions. Here we only consider the average transmission delay under the worst and the ideal traffic conditions. The worst case occurs when all of the neighbors of a specific node transmit data packets in all their assigned slots and each of them causes $k$ collisions in a frame. The ideal case occurs when all of the neighbors of a specific node do not contribute any collisions to its transmissions because either they have no packet to transmit or their TSAF's have no common roots with the TSAF of this node.

Definition 8: The average transmission delay under the worst traffic condition is called the maximum transmission delay $D T_{\max }$. It is defined as the ratio of the frame length to the minimum number of successful transmission slots in a frame, i.e.,

$$
\begin{aligned}
D T_{\max }= & (\text { frame length }) /(\text { minimum number of } \\
& \text { successful transmissions in a frame }) \\
= & L / T_{\min } .
\end{aligned}
$$

Definition 9: The average transmission delay under the ideal traffic condition is called the minimum transmission delay $D T_{\text {min }}$. It is defined as the ratio of the frame length to the maximum number of successful transmission slots in a frame, i.e.,

$$
\begin{aligned}
D T_{\min }= & (\text { frame length }) /(\text { maximum number of } \\
& \text { successful transmissions in a frame }) \\
= & L / T_{\max } .
\end{aligned}
$$

$D T_{\max }$ and $D T_{\min }$ are the upper and lower bounds of the average transmission delay, respectively.

Theorem 4: $D T_{\max }$ is equal to $p^{2} /\left(p-k D_{\max }\right)$, where $D_{\max }$ is the maximum degree in the network, $k$ is the maximum degree of TSAF's, and $p$ is the number of assigned slots in each frame for each node.

Proof: The frame length $L$ is equal to $p^{2}$. From (14), we get

$$
D T_{\max }=L / T_{\min }=p^{2} / T_{\min }=p^{2} /\left(p-k D_{\max }\right) .
$$

This $D T_{\max }$ is the average longest time between two successive successful transmissions. Similarly, we can get the following theorem for $D T_{\min }$ which is the average shortest time between two successive successful transmissions.

Theorem 5: $D T_{\min }$ is equal to $p$, where $p$ is the number of assigned slots in each frame for each node. 
Proof: From (15), we get

$$
D T_{\min }=L / T_{\max }=p^{2} / p=p .
$$

So, the average transmission delay will be between $p^{2} /(p-$ $\left.k D_{\max }\right)$ and $p$. Furthermore, we can get the following conclusion.

Theorem 6: The difference between $D T_{\max }$ and $D T_{\min }$ of our optimal algorithm is no more than $2 k D_{\max }+1$.

Proof: From (16) and (17), we get

$$
\begin{aligned}
D T_{\max }-D T_{\min } & =\left(p^{2} / T_{\min }\right)-p \\
& =\left[p^{2} /\left(p-k D_{\max }\right)\right]-p .
\end{aligned}
$$

From Theorem 3, the optimal value of $p$ is the prime number closest to

$$
\left\{\begin{array}{l}
2 k D_{\max }, \quad \text { if } N^{1 /(k+1)} \leq 2 k D_{\max } \\
N^{1 /(k+1)}, \quad \text { otherwise. }
\end{array}\right.
$$

Substituting the above $p$ value into (18), we will get

$$
\left\{\begin{array}{l}
D T_{\max }-D T_{\min } \leq 2 k D_{\max }+1 \\
\quad \text { if } N^{1 /(k+1)} \leq 2 k D_{\max } \\
k D_{\max } \leq D T_{\max }-D T_{\min } \leq 2 k D_{\max } \\
\text { otherwise. }
\end{array}\right.
$$

Hence, we conclude that $D T_{\max }-D T_{\min }$ is no more than $2 k D_{\max }+1$.

Theorem 7: For a given $k, G_{\min }$ of our algorithm is larger than the guaranteed throughput of conventional TDMA fixed assignment scheduling algorithm when $4 k D_{\max } \leq N$.

Proof: From (5), we know

$$
G_{\min }=T_{\min } / L=T_{\min } / p^{2}=1 /\left(p^{2} / T_{\min }\right)
$$

If $G_{\min } \geq 1 / N$, then

$$
\begin{aligned}
1 /\left(p^{2} / T_{\min }\right) & \geq 1 / N \\
N T_{\min } & \geq p^{2} \\
N\left(p-k D_{\max }\right) & \geq p^{2} \\
0 & \geq p^{2}-N p+N k D_{\max } .
\end{aligned}
$$

Solving the above inequality, we get

$$
\frac{N-\sqrt{N^{2}-4 N k D_{\max }}}{2} \leq p \leq \frac{N+\sqrt{N^{2}-4 N k D_{\max }}}{2}
$$

when

$$
\left(N^{2}-4 N k D_{\max }\right) \geq 0, \quad N \geq 4 k D_{\max }
$$

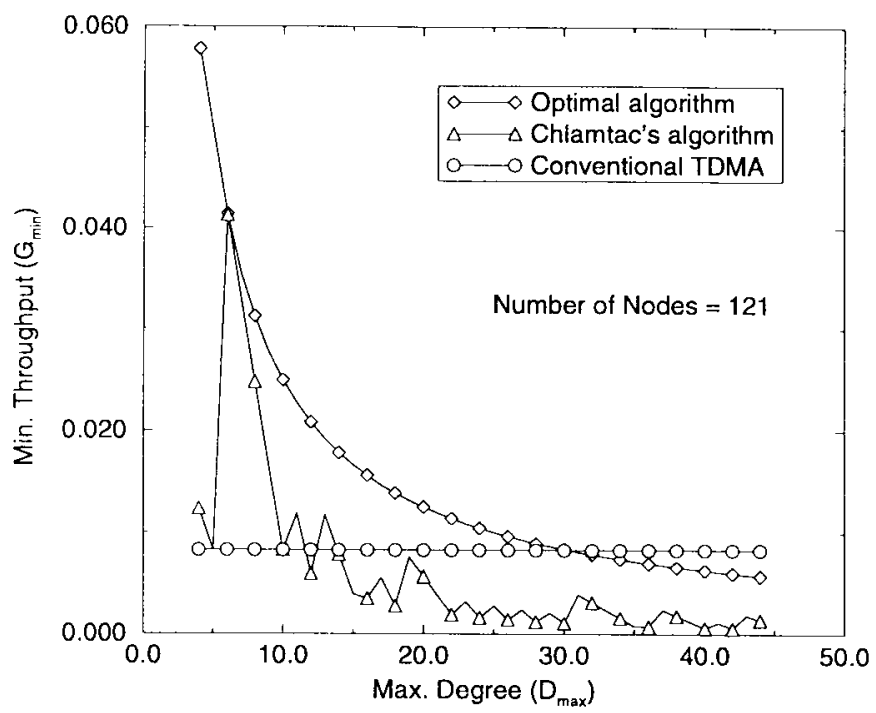

Fig. 2. The guaranteed throughput $G_{\min }$ for $N=121$.

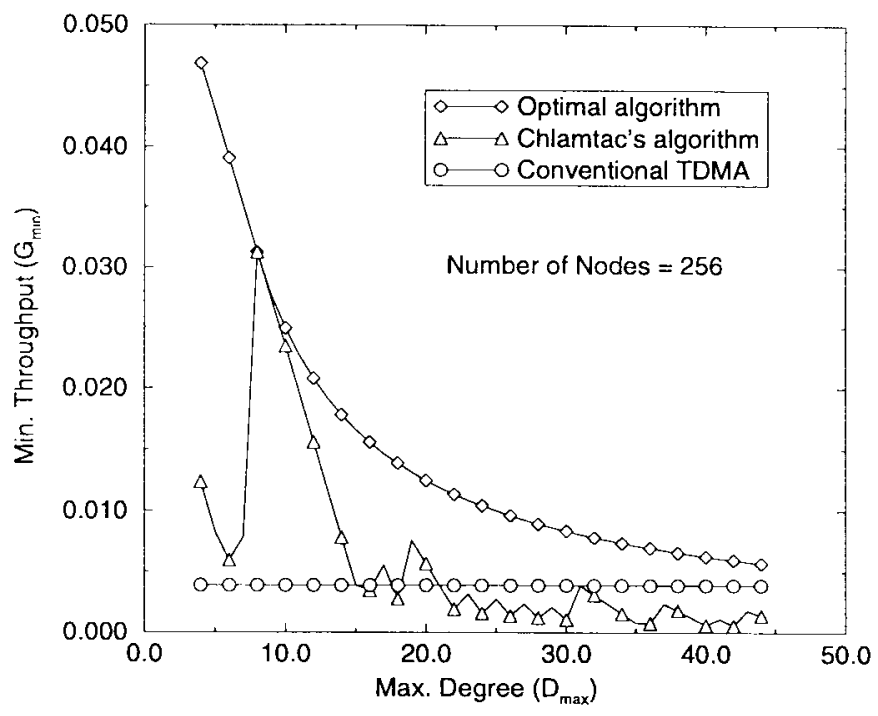

Fig. 3. The guaranteed throughput $G_{\min }$ for $N=256$.

\section{NumERICAL RESUlTS AND DisCUSSION}

In this section we quantitatively compare the above optimal scheduling algorithm with the conventional TDMA fixed assignment scheme and the one proposed by Chlamtac and Farago [2]. For convenience we refer to these three algorithms as the optimal, the conventional TDMA, and Chlamtac's algorithms, respectively. We considered four cases where the number of nodes in the network $N$ is $121,256,800$, and 1024, respectively, and the range of the maximum degree $D_{\max }$ is from 4 to 44 . The bigger the value of $D_{\max }$, the higher the density of mobile nodes in a certain area. For each given $N$ and $D_{\max }, G_{\min }, D T_{\max }$, and $D T_{\min }$ are obtained for each of the scheduling algorithms. $G_{\min }$ gives the minimum throughput of the network. $D T_{\max }$ and $D T_{\min }$ give the upper and lower bounds of the average transmission delay for a packet.

Figs. 2-5 show $G_{\min }$ for the four cases. They show that our optimal algorithm has the best performance. It always performs much better than Chlamtac's algorithm, especially 


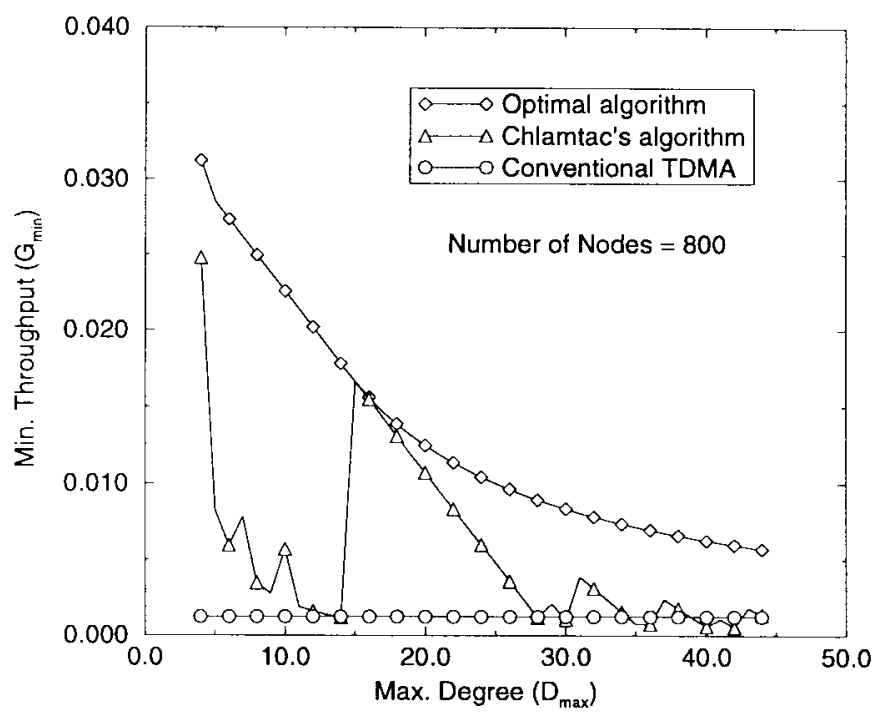

Fig. 4. The guaranteed throughput $G_{\min }$ for $N=800$.

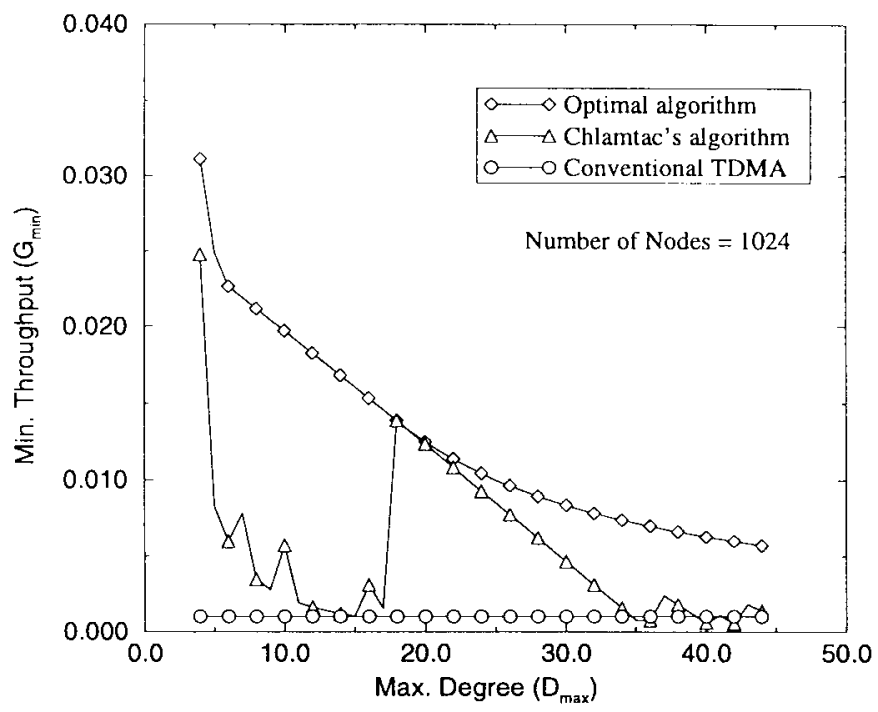

Fig. 5. The guaranteed throughput $G_{\min }$ for $N=1024$.

when the value of the maximum number of degree is not very high. For the case of $N=121$ and $D_{\max }=5, G_{\min }$ of the optimal algorithm is six times better than $G_{\min }$ of Chlamtac's algorithm. $G_{\min }$ of Chlamtac's algorithm fluctuates with $D_{\max }$ because it only focuses on minimizing the frame length. If the actual value of $D_{\max }$ deviates from the design value of $D_{\max }$, the performance may degrade drastically. In our algorithm the performance is not very sensitive to this deviation.

Due to the fixed assignment property, $G_{\min }$ of conventional TDMA is a flat line. When $N$ is large, Chlamtac's algorithm performs better than conventional TDMA in most cases. If $N$ is small, then Chlamtac's algorithm performs better only when $D_{\max }$ is sufficiently small. Our optimal algorithm performs better than conventional TDMA for most of the $D_{\max }$ values for all cases. This agrees with Theorem 7 derived in the previous section.

Figs. 6-9 show $D T_{\min }$ and $D T_{\max }$ for the four cases. They show that Chlamtac's algorithm has the lowest $D T_{\min }$ and our

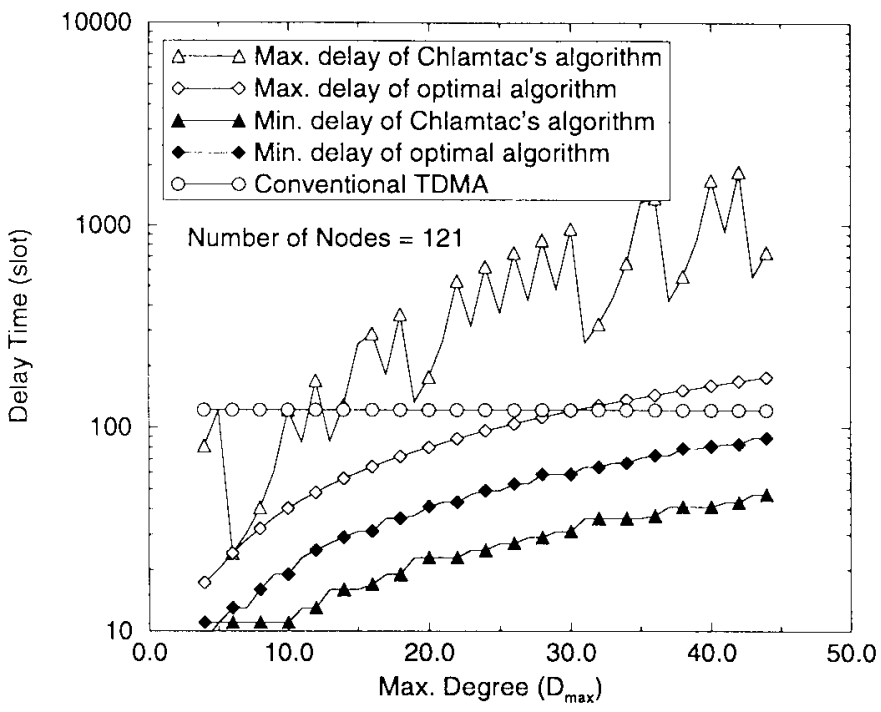

Fig. 6. The scheduling delays for $N=121$.

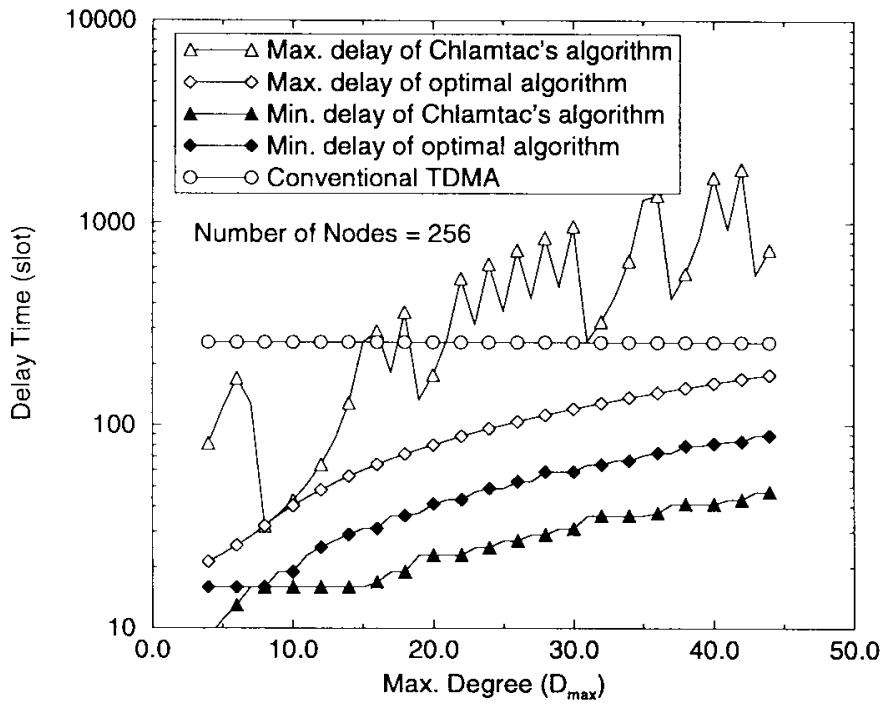

Fig. 7. The scheduling delays for $N=256$.

$D T_{\min }$ is similar. Chlamtac's algorithm also has the highest $D T_{\max }$ when $N$ is small or $D_{\max }$ is large. Its $D T_{\max }$ also fluctuates a lot with $D_{\max }$. Our $D T_{\max }$ increases smoothly with $D_{\max }$ and is much less than $D T_{\max }$ of Chlamtac's algorithm. $D T_{\min }$ and $D T_{\max }$ of our optimal algorithm are almost always less than the average transmission delay of conventional TDMA. By comparing the differences of $D T_{\max }$ and $D T_{\min }$, we see that the optimal algorithm has a smaller transmission delay variation than Chlamtac's algorithm. The small transmission delay variation implies that our algorithm is more stable, which is an important preferred feature in network design.

In Chlamtac's algorithm the number of total assigned slots for each node in a frame $p$ is a power of prime, i.e., $p=q^{m}$, where $q$ is a prime number and $m$ is a positive integer $(\geq 1)$. In their papers [2], [3] they did not provide an explicit method to calculate the positions of the assigned transmission slots when $m>1$ because the calculation based on finite field will be 


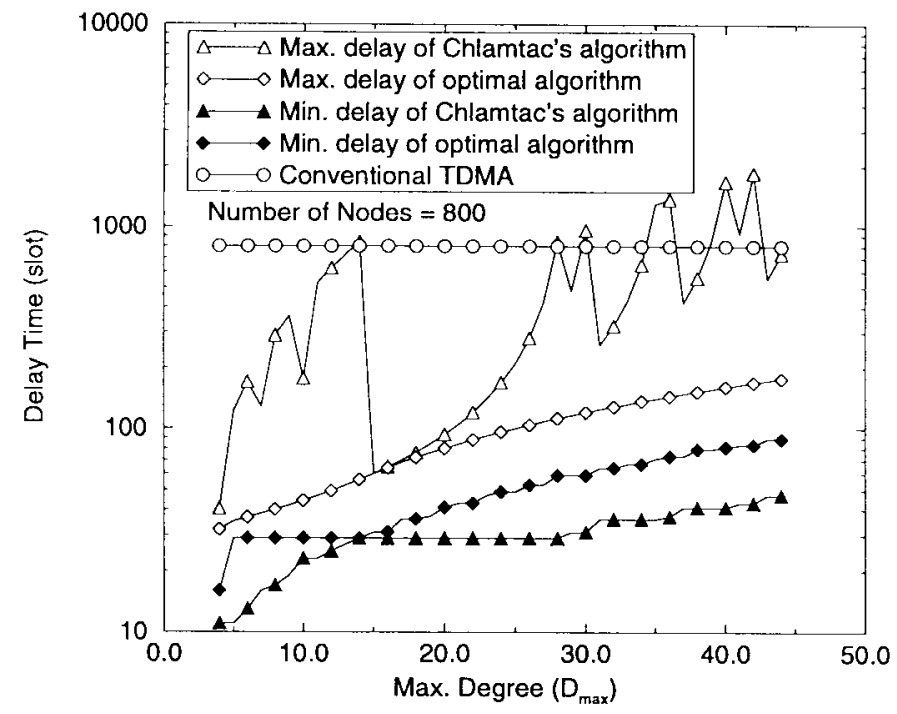

Fig. 8. The scheduling delays for $N=800$.

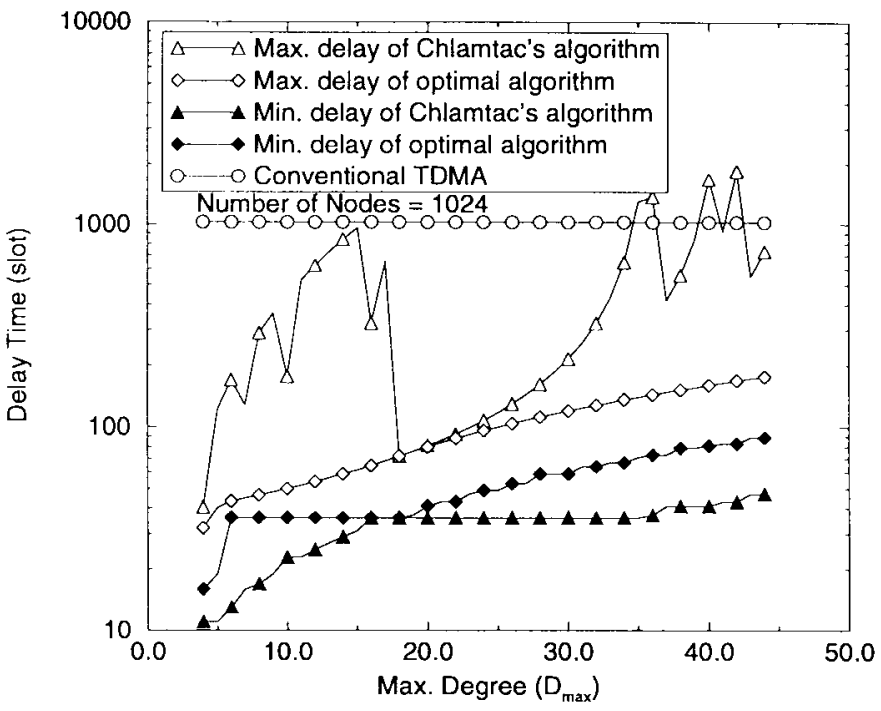

Fig. 9. The scheduling delays for $N=1024$.

more complicated. (Using the geometric mapping method, this problem can be solved by the algorithm in [13].) For ease of implementation, in this paper our algorithm will only consider the cases when $p$ is equal to a prime number. Although this restriction will limit the choices of $p$, we found that the performance of our algorithm is almost the same for $p$ a prime and $p$ a power of prime.

In Figs. 10 and 11 we investigate the effect of inaccuracies in the estimation of $D_{\max }$ on the minimum throughput $G_{\min }$. In Fig. 10 we choose $N=121$ and $D_{\max }=10$ as the design values for our algorithm, then we find that the corresponding optimal values of $p$ and $k$ are 19 and 1, which provide the maximal minimum throughput. When the actual value of $D_{\max }$ varies from the design value of ten, the optimal values of $p, k$ and maximal minimum throughput may change. The black bars in the figures represent the actual $G_{\min }$ that the network experiences when $N=121$ and the actual $D_{\max }$ is equal

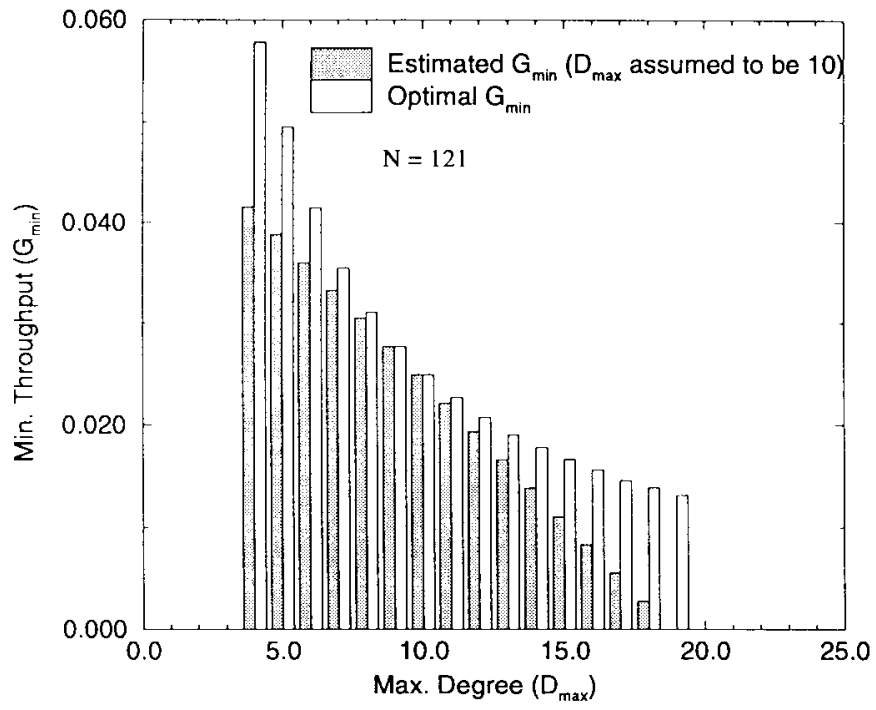

Fig. 10. $G_{\min }$ as functions of $D_{\max }$ when $p$ varies and for $p=19$, respectively.

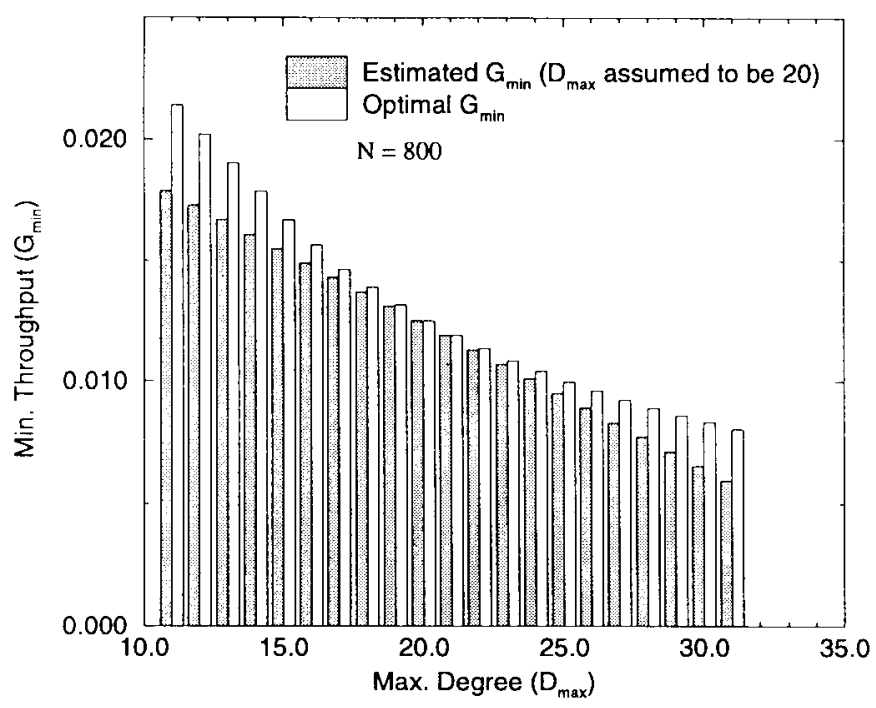

Fig. 11. $G_{\min }$ as functions of $D_{\max }$ when $p$ varies and for $p=41$, respectively.

to the value shown on the $x$ axis (but using $N=121$ and $D_{\max }=10$ as the design values). The white bars in the same figure represent the maximal $G_{\min }$ that the network should have if we choose $N=121$ and $D_{\max }=$ the value shown on the $x$ axis as design parameters. The penalty on $G_{\min }$, i.e., the difference between the white bar and the black bar, is caused by the differences of their optimal $p$ and $k$ values. The figure shows that the penalty on $G_{\min }$ caused by choosing suboptimal values of $p$ and $k$ is very small when the actual $D_{\max }$ is close to the design value of $D_{\max }(=10)$. As the difference between the actual $D_{\max }$ and the design value of $D_{\max }$ increases, the penalty on $G_{\min }$ also increases. We observe similar behavior in Fig. 11, but note that the rate of increase in the penalty on $G_{\min }$ is much slower (compared with Fig. 10). So we conclude that the minimum throughput $G_{\min }$ is not very sensitive to the accuracy of estimated $D_{\max }$, and the effect becomes smaller when the size of the network increases. 


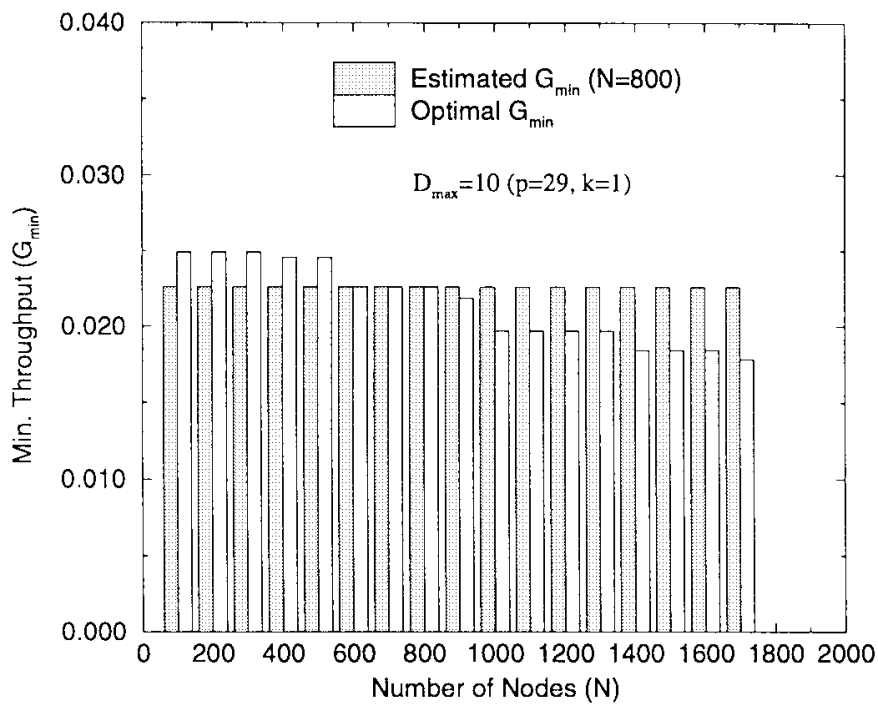

Fig. 12. $G_{\min }$ as a function of the number of nodes $N$ for fixed $D_{\max }(=10)$.

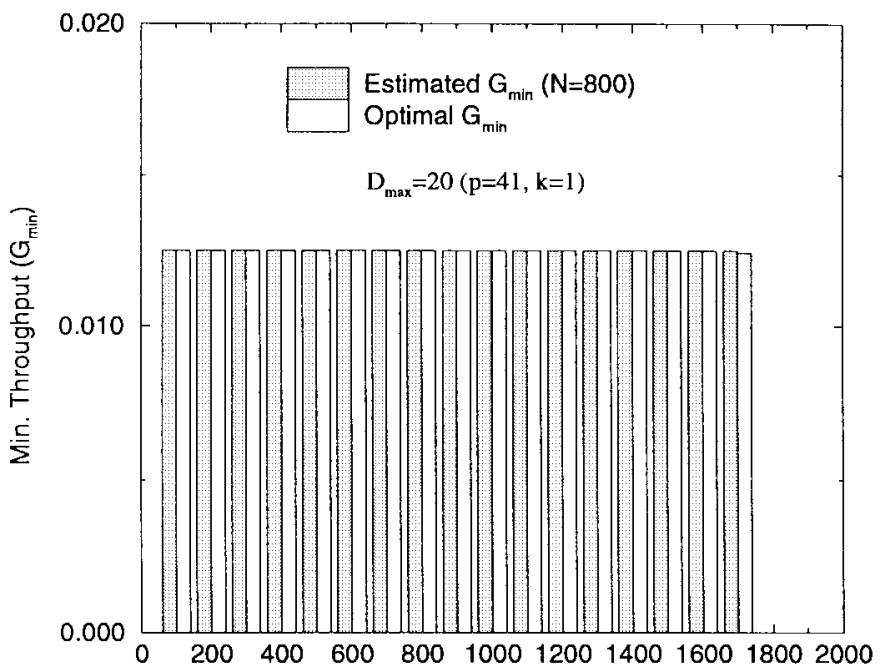

Fig. 13. $G_{\min }$ as a function of the number of nodes $N$ for fixed $D_{\max }(=20)$.

Figs. 12 and 13 consider the effect of inaccuracies in the estimation of $N$ on the minimum throughput $G_{\min }$. In Fig. 12 we use $N=800$ and $D_{\max }=10$ as our design values and find the corresponding optimal values of $p$ and $k$ are 29 and 1 . The optimal values of $p, k$ and the maximal minimum throughput are expected to change when the actual $N$ varies from the design value of 800 . The white bars in the figures represent the optimal $G_{\min }$ that the network will have if we choose $D_{\max }=10$ and $N$ is equal to the value shown on the $x$ axis as design parameters. The black bars represent the actual $G_{\min }$, i.e., the estimated $G_{\min }$, of the network when $D_{\max }=10$ and $N=800$ are used as the design values. Although the estimated $G_{\min }$ is better than the optimal $G_{\min }$ when the actual value of $N$ exceeds $841=29^{2}$, i.e., the inequality stated in (3) is violated, the minimum throughput will be zero for some nodes because the total number of TSAF's is less than the total number of nodes. In this situation either some nodes will not be assigned a TSAF or some nodes will share the same
TSAF. If the nodes with the same TSAF are adjacent to each other, their minimum throughput becomes zero because their transmissions may potentially collide all of the time. When the actual value of $N$ is less than or equal to 841, there is a penalty on $G_{\min }$ which increases with the difference in the actual value of $N$ and the design value of $N$. Similar results can be found in Fig. 13, but the rate of increase in the penalty on $G_{\min }$ is much slower (compared with Fig. 12). This proves that the minimum throughput $G_{\min }$ of our algorithm is not very sensitive to the accuracy in the estimation of $N$, and the effect becomes smaller when the network size is large.

\section{CONCLUSIONS}

In a multihop TDMA network, transmission scheduling has a very important impact on the performance. A scheduling algorithm which is topology-transparent is particularly desirable when the network is highly mobile. In this paper, following the approach in [2] but with a different design strategy, we have proposed an optimal transmission scheduling algorithm to provide the maximal minimum throughput. This algorithm is easy to implement and suited for distributed implementation because it only needs global information about the number of nodes $N$ and the maximum degree $D_{\max }$ in the network. The transparency to topology changes is inherent in the algorithm and no dynamic control of algorithm parameters is required.

We also derived the minimum throughput, the minimum delay time (when the system is under very light traffic), and the maximum delay time (when the system is under very heavy traffic) for the scheduling algorithm. Our algorithm is shown to be optimal because it maximizes the minimum throughput. The optimal minimum throughput is also insensitive to inaccuracies in the estimated design values $N$ and $D_{\max }$.

Compared with the conventional TDMA fixed scheduling algorithm and Chlamtac's algorithm, our algorithm performs best.

\section{REFERENCES}

[1] D. J. Baker and A. Ephremides, "The architectural organization of a mobile radio network via a distributed algorithm," IEEE Trans. Commun., vol. COM-29, pp. 1694-1701, Nov. 1981.

[2] I. Chlamtac and A. Farago, "Making transmission schedules immune to topology changes in multi-hop packet radio networks," IEEE/ACM Trans. Networking, vol. 2, pp. 23-29, Feb. 1994.

[3] I. Chlamtac, A. Farago, and H. Y. Ahn, "A topology transparent link activation protocol for mobile CDMA radio networks," IEEE J. Select. Areas Commun., vol. 12, pp. 1426-1433, Oct. 1994.

[4] A. M. Chou and V. O. K. Li, "Slot allocation strategies for TDMA protocols in multihop packet radio networks," in IEEE INFOCOM '92, Florence, Italy, May 1992, pp. 710-716.

[5] I. Cidon and M. Sidi, "Distributed assignment algorithms for multihop packet radio networks," IEEE Trans. Comput., vol. 38, pp. 1351-1361, Oct. 1989.

[6] U. Dudley, Elementary Number Theory. San Francisco, CA: Freeman, 1969.

[7] A. Ephremides and T. V. Truong, "Scheduling broadcasts in multihop radio networks," IEEE Trans. Commun., vol. 38, pp. 456-460, Apr. 1990.

[8] A. Farago, I. Chlamtac, and H. Y. Ahn, "Nearly optimum scheduling in mobile CDMA packet radio networks," in IEEE MILCOM'92, San Diego, CA, Oct. 1992, pp. 769-773.

[9] B. Hajek and G. Sasaki, "Link scheduling in polynomial time," IEEE Trans. Inform. Theory, vol. 34, pp. 910-917, Sept. 1988.

[10] T. Hou and V. O. K. Li, "Transmission range control in multihop packet radio networks," IEEE Trans. Commun., vol. COM-34, pp. 38-44, Jan. 1986 
[11] L. Hu, "A novel topology control for multihop packet radio networks," in IEEE INFOCOM '91, Miami, FL, Apr. 1991, pp. 1084-1093.

[12] K. Hung and T. Yum, "Fair and efficient transmission scheduling in multihop packet radio networks," in IEEE GLOBECOM '92, Nov. 1992, pp. 6-10.

[13] J. H. Ju and V. O. K. Li, "Latin squares for multichannel scheduling design," submitted for publication.

[14] L. Kleinrock and J. Silvester, "Optimum transmission radii for packet radio networks or why six is a magic number," in Natl. Telecommunication Conf., Birmingham, AL, Dec. 1978, pp. 4.3.1-5.

[15] R. Ramaswami and K. K. Parhi, "Distributed scheduling of broadcasts in a radio network," in IEEE INFOCOM '89, Ottawa, Ont., Canada, Apr. 1989, pp. 497-504.

[16] L. Tassiulas and A. Ephremides, "Jointly optimal routing and scheduling in packet radio networks," IEEE Trans. Inform. Theory, vol. 38, pp. 165-168, Jan. 1992.

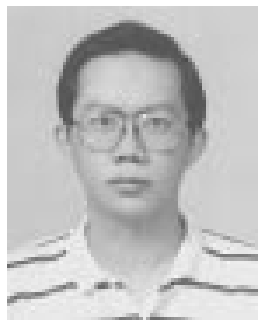

Ji-Her Ju (S'92-M'96) was born in Taiwan, R.O.C., in 1961. He received the B.S. and M.S. degrees from National Cheng Kung University, Tainan, Taiwan, R.O.C., in 1984 and 1986, respectively, and the Ph.D. degree from University of Southern California, Los Angeles, in 1996, all in electrical engineering.

Since 1986 he has been with the Communication Section, Chung Shan Institute of Science and Technology (CSIST), Taiwan, R.O.C., where he is an Associate Researcher. His research interests are in the areas of mobile communication networks, personal communication systems, broad-band networks and multimedia communications, especially in the network architecture design, network management, network protocol design, and performance evaluation.

Dr. Ju coauthored a paper that received the Best Student Paper Award at the 4th Annual Networld+Interop Engineer Conference, 1997, cosponsored by the IEEE Communications Society.

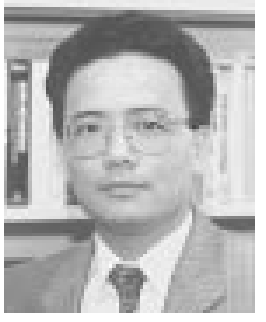

Victor O. K. Li (S'80-M'81-SM'86-F'92) was born in Hong Kong in 1954. He received the S.B., S.M., and Sc.D. degrees in electrical engineering and computer science from the Massachusetts Institute of Technology, Cambridge, MA, in 1977, 1979, and 1981, respectively.

He joined the University of Southern California (USC), Los Angeles, in February 1981, and became a Professor of electrical engineering and the Director of the USC Communication Sciences Institute. Since September 1997 he has been with the University of Hong Kong, Hong Kong, where he is the Chair Professor of information engineering in the Department of Electrical and Electronic Engineering, and the Managing Director of Versitech, Ltd., the university contract research and consulting company. His research interests include high-speed communication networks, personal communication networks, and distributed multimedia systems. He has published over 200 technical articles and has lectured and consulted extensively around the world. He was a Distinguished Lecturer at the University of California at San Diego, the National Science Council of Taiwan, and the California Polytechnic Institute. $\mathrm{He}$ has given keynote addresses and served on the advisory boards of numerous international conferences.

Prof. Li chaired the Computer Communications Technical Committee of the IEEE Communications Society from 1987 to 1989, and the Los Angeles Chapter of the IEEE Information Theory Group from 1983 to 1985 . He chaired the Steering Committee of the International Conference on Computer Communications and Networks $\left(I C^{3} N\right)$ from 1992 to 1997 and served as General Chair of the 1st Annual $I C^{3} N$, June 1992, as Technical Program Chair of the Institution of Electrical Engineers (IEE) Personal Communication Services Symposium, June 1995, and as Chair of the 4th IEEE Workshop on Computer Communications, October 1989. He has served as an Editor of Network and Telecommunication Systems, Guest Editor of the IEEE JouRnaL on Selected AREas in Communications and Computer Networks and ISDN Systems, and is now serving as an Editor of ACM Wireless Networks. 